\title{
INTERACTION OF SOME MEROMORPHIC SOLUTIONS OF THE KdV EQUATION
}

\author{
by M. KOVALYOV and K. M. LEE \\ (Received 15th May 1997)
}

\begin{abstract}
A necessary and sufficient condition for confluence of two poles of a class of meromorphic solutions of the $\mathrm{KdV}$ equation is introduced and proved.
\end{abstract}

1991 Mathematics subject classification: 35Q51, 35 Q53.

\section{Introduction and statement of the main result}

In this paper we study interaction of some meromorphic solutions of the Korteweg-de Vries equation

$$
u_{t}-6 u u_{x}+u_{x x x}=0, \quad \lim _{x \rightarrow \pm \infty} u(t, x)=0 .
$$

These solutions, sometimes referred to as positions $[11,12,14,15]$, and sometimes as harmonic breathers [7] are of the form

$$
u=\frac{8 \ell^{2} \sin 2 \ell\left(4 \ell^{2} t+x-\gamma\right)}{\sin 2 \ell\left(4 \ell^{2} t+x-\gamma\right)-2 \ell\left(12 \ell^{2} t+x-p\right)}+8 \ell^{2}\left[\frac{1-\cos 2 \ell\left(4 \ell^{2} t+x-\gamma\right)}{\sin 2 \ell\left(4 \ell^{2} t+x-\gamma\right)-2 \ell\left(12 \ell^{2} t+x-p\right)}\right]^{2} .
$$

Probably first studied in $[11,12,14,15],(1.2)$ naturally arises when one generates explicit solutions of (1.1) by means of the Darboux transform [11, 12] or attempts to define solutions of (1.1) with the "simplest continuous spectrum" [7].

Solutions (1.2) possess a pole whose location depends on time. This pole may play role of a "centre" of the corresponding harmonic breather in much the same way as the local minimum of the soliton solution $-\frac{2 \ell^{2}}{\cosh ^{2} \ell\left(x-4 \ell^{2} t-\varphi\right)}$ plays that role for the corresponding soliton. The solution (1.2) can be written as

$$
\begin{aligned}
& u(t, x)=-2 \frac{\partial^{2}}{\partial x^{2}} \ln \tau(t, x) \\
& \tau(t, x)=\frac{\sin 2 \ell\left(4 \ell^{2} t+x-\gamma\right)}{2 \ell}-\left(12 \ell^{2} t+x-p\right)
\end{aligned}
$$


so the pole of (1.2) is exactly the zero of the $\tau$-function in (1.3) and the study of motion of the pole of (1.2) can be reduced to the study of motion of the zero of $\tau(t, x)$. Since $\tau^{\prime}(t, x)=\frac{\partial \tau(t, x)}{\partial x}=\cos 2 \ell\left(4 \ell^{2} t+x-\gamma\right)-1 \leq 0$, the $\tau$-function itself is monotonically decreasing in $x$ from $+\infty$ to $-\infty$ and thus always has exactly one zero. The zero is simple unless

$$
2 \ell\left(4 \ell^{2} t+x-\gamma\right)=2 \pi n, \quad n \in \mathbb{I}
$$

and

$$
12 \ell^{2} t+x-p=0
$$

in which case the zero is of third order. The solution of (1.4) is of the form:

$$
t=\frac{p-\gamma}{8 \ell^{2}}+\frac{\pi n}{4 \ell^{3}}, \quad x=\frac{3 \gamma-p}{2}-\frac{3 \pi n}{\ell}, \quad n \in \mathbb{N}
$$

Such points are often referred to as resonances [14].

We can define superposition of two harmonic breathers $[7,13]$ and study their interaction in a way similar to that for solitons. Due to the complicated form of the two-harmonic-breather solution, the interaction of the harmonic breathers when they are close to each other has, so far, been studied only numerically [17]. Here we obtain some analytical results similar to those of $[2,5,9,10,13,16]$ for solitons.

To do this we use the following representation of the two-harmonic-breather solution obtained in [7]:

$$
w(t, x)=-2 \frac{\partial^{2}}{\partial x^{2}} \ln \tau(t, x), \quad \tau(t, x)=\tau_{1}(t, x) \tau_{2}(t, x)-q^{2}(t, x)
$$

where

$$
\begin{gathered}
\tau_{i}(t, x)=\frac{\sin 2 \ell_{i} \xi_{i}}{2 \ell_{i}}-\eta_{i}, \quad \xi_{i}=x+4 \ell_{i}^{2} t-\gamma, \quad \eta_{i}=x+12 \ell_{i}^{2} t-p_{i}, \quad i=1,2 ; \\
q=\frac{\sin \left(\ell_{1} \xi_{1}-\ell_{2} \xi_{2}\right)}{\ell_{1}-\ell_{2}}-\frac{\sin \left(\ell_{1} \xi_{1}+\ell_{2} \xi_{2}\right)}{\ell_{1}+\ell_{2}}=\frac{2}{\ell_{1}^{2}-\ell_{2}^{2}}\left(\ell_{2} \sin \ell_{1} \xi_{1} \cos \ell_{2} \xi_{2}-\ell_{1} \cos \ell_{1} \xi_{1} \sin \ell_{2} \xi_{2}\right), \\
\ell_{1} \neq \ell_{2}, \quad \ell_{1}>0, \quad \ell_{2}>0 .
\end{gathered}
$$

The analytical results are summarized in the following theorem.

Theorem. (a) The $\tau$-function of (1.6) always has at most two zeros, i.e. for each value of $t \in \mathbb{R}$, there are at most two values of $x$ satisfying $\tau(t, x)=0$.

(b) Two distinct roots of $\tau(t, x)=0$, which we denote by $x_{1}(t)$ and $x_{2}(t)$, merge into one for some value of $t$ if and only if the quantities 


$$
\begin{aligned}
& n_{1}=\frac{2 \ell_{1}}{3 \pi\left(\ell_{2}^{2}-\ell_{1}^{2}\right)}\left[6 \ell_{2}^{2}\left(p_{1}-\gamma_{1}\right)-6 \ell_{1}^{2}\left(p_{2}-\gamma_{2}\right)-\ell_{1}^{2}\left(3 \gamma_{2}-p_{2}-3 \gamma_{1}+p_{1}\right)\right] \\
& n_{2}=\frac{2 \ell_{2}}{3 \pi\left(\ell_{1}^{2}-\ell_{2}^{2}\right)}\left[6 \ell_{1}^{2}\left(p_{2}-\gamma_{2}\right)-6 \ell_{2}^{2}\left(p_{1}-\gamma_{1}\right)-\ell_{2}^{2}\left(3 \gamma_{1}-p_{1}-3 \gamma_{2}+p_{2}\right)\right]
\end{aligned}
$$

are integers and are either both even or both odd. If $n_{1}$ and $n_{2}$ are both even, then at time $t=\frac{p_{2}-\gamma_{2}}{8 \ell_{2}^{2}}+\frac{\pi n_{2}}{16 c_{2}^{3}}=\frac{p_{1}-p_{1}}{8 \ell_{1}^{2}}+\frac{\pi n_{1}}{16 t_{1}^{3}}, \tau(t, x)$ has a single root $x=\frac{3 \eta_{1}-p_{1}}{2}-\frac{3 \pi n_{1}}{4 t_{1}}=\frac{3 y_{2}-p_{2}}{2}-\frac{3 \pi n_{2}}{4 \ell_{2}}$ of order

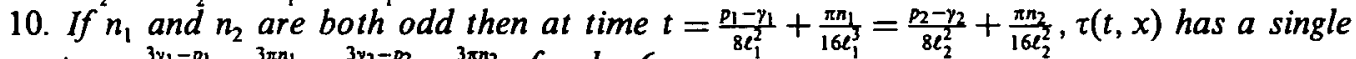
root $x=\frac{3 y_{1}-p_{1}}{2}-\frac{3 \pi n_{1}}{4 \ell_{1}}=\frac{3 y_{2}-p_{2}}{2}-\frac{3 \pi n_{2}}{4 \ell_{2}}$ of order 6 .

\section{Proof of the Theorem}

We break up the proof into a sequence of lemmas.

Lemma 1. The $\tau$-function defined in (1.6) and its components satisfy the following identities:

$$
\begin{aligned}
\tau & =\tau_{1} \tau_{2}-q^{2} \\
\tau_{i} & =\frac{\sin 2 \ell_{i} \xi_{i}}{2 \ell_{i}}-\eta_{i}, \quad \xi_{i}=x+4 \ell_{i}^{2} t-\gamma_{i}, \quad \eta_{i}=x+12 \ell_{i}^{2} t-p_{i}, \quad i=1,2 \\
q & =\frac{2}{\ell_{1}^{2}-\ell_{2}^{2}}\left(\ell_{2} \sin \ell_{1} \xi_{1} \cos \ell_{2} \xi_{2}-\ell_{1} \cos \ell_{1} \xi_{1} \sin \ell_{2} \xi_{2}\right) \\
q^{\prime} & =\frac{\partial q}{\partial x}=2 \sin \ell_{1} \xi_{1} \sin \ell_{2} \xi_{2} \\
q^{\prime \prime} & =\frac{\partial^{2} q}{\partial x^{2}}=2 \ell_{1} \cos \ell_{1} \xi_{1} \sin \ell_{2} \xi_{2}+2 \ell_{2} \sin \ell_{1} \xi_{1} \cos \ell_{2} \xi_{2} \\
\tau_{i}^{\prime} & =\frac{\partial \tau_{i}}{\partial x}=\cos 2 \ell_{i} \xi_{i}-1=-2 \sin ^{2} \ell_{i} \xi_{i}, \quad i=1,2 \\
\tau_{i}^{\prime \prime} & =\frac{\partial^{2} \tau_{i}}{\partial x^{2}}=-4 \ell_{i} \sin _{1} \ell_{i} \xi_{i} \cos \ell_{i} \xi_{i}, \quad i=1,2 \\
\tau^{\prime} & =\frac{\partial \tau}{\partial x}=-2 \tau_{1} \sin ^{2} \ell_{2} \xi_{2}-2 \tau_{2} \sin ^{2} \ell_{1} \xi_{1}-2 q \sin \ell_{1} \xi_{1} \sin \ell_{2} \xi_{2}
\end{aligned}
$$




$$
\begin{aligned}
\tau^{\prime \prime}= & \frac{\partial^{2} \tau}{\partial x^{2}}=-2 \ell_{1} \tau_{2} \sin 2 \ell_{1} \xi_{1}-2 \ell_{2} \tau_{1} \sin 2 \ell_{2} \xi_{2} \\
& \quad-\frac{8}{\ell_{1}^{2}-\ell_{2}^{2}}\left(\ell_{2}^{2} \sin ^{2} \ell_{1} \xi_{1} \cos ^{2} \ell_{2} \xi_{2}-\ell_{1}^{2} \sin ^{2} \ell_{2} \xi_{2} \cos ^{2} \ell_{1} \xi_{1}\right) \\
\tau^{\prime \prime \prime}= & -4 \ell_{1}^{2} \tau_{2} \cos 2 \ell_{1} \xi_{1}-4 \ell_{2}^{2} \tau_{1} \cos 2 \ell_{2} \xi_{2}+\frac{4 \ell_{2}}{\ell_{1}^{2}-\ell_{2}^{2}} \sin 2 \ell_{2} \xi_{2}\left(\ell_{1}^{2}+\ell_{1}^{2} \cos ^{2} \ell_{1} \xi_{1}+\ell_{2}^{2} \sin ^{2} \ell_{1} \xi_{1}\right) \\
& -\frac{4 \ell_{1}}{\ell_{1}^{2}-\ell_{2}^{2}} \sin 2 \ell_{1} \xi_{1}\left(\ell_{2}^{2}+\ell_{2}^{2} \cos ^{2} \ell_{2} \xi_{2}+\ell_{1}^{2} \sin ^{2} \ell_{2} \xi_{3}\right) \\
\tau= & -\frac{\tau^{\prime} \tau_{1}}{2 \sin ^{2} \ell_{1} \xi_{1}}-\left(\frac{\sin \ell_{2} \xi_{2}}{\sin \ell_{1} \xi_{1}} \tau_{1}+q\right)^{2} \\
\tau= & -\frac{\tau^{\prime} \tau_{2}}{2 \sin ^{2} \ell_{2} \xi_{2}}-\left(\frac{\sin \ell_{1} \xi_{1}}{\sin \ell_{2} \xi_{2}} \tau_{2}+q\right)^{2}
\end{aligned}
$$

Proof of (2.1)-(2.10) is by direct computations. Equation (2.11a) is obtained by solving (2.8) for $\tau_{2}$ and then substituting $\tau_{2}=-\frac{\tau^{\prime}}{2 \sin ^{2} \ell_{1} \xi_{1}}-\frac{\sin ^{2} \ell_{2} \xi_{2}}{\sin ^{2} \ell_{1} \xi_{1}} \tau_{1}-\frac{2 q \sin \ell_{2} \xi_{2}}{\sin \ell_{1} \xi_{1}}$ into (2.1); $(2.11 \mathrm{~b})$ is obtained in a similar manner.

Lemma 2. If $\tau(t, x)=\tau^{\prime}(t, x)=0$, then $\tau^{\prime \prime}(t, x)=0$.

Proof. If neither $\sin \ell_{1} \xi_{1}=0$ nor $\sin \ell_{2} \xi_{2}=0$, (2.11) gives us $\tau_{1}=-\frac{\sin \ell_{1} \xi_{1}}{\sin \ell_{2} \xi_{2}} q$, $\tau_{2}=-\frac{\sin \ell_{2} \xi_{2}}{\sin \ell_{1} \xi_{1}} q$. Substituting these into (2.9) we obtain $\tau^{\prime \prime}=0$. If $\sin \ell_{1} \xi_{1}=0\left(\sin \ell_{2} \xi_{2}=0\right.$ is handled in exactly the same manner), then $\tau^{\prime}=0$ and (2.8) implies $\tau_{1} \sin \ell_{2} \xi_{2}=0$ and therefore either $\sin \ell_{2} \xi_{3}=0$ or $\tau_{1}=0$. If $\sin \ell_{2} \xi_{2}=0$ then substituting $\sin \ell_{1} \xi_{1}=$ $\sin \ell_{2} \xi_{2}=0$ into (2.9) we obtain $\tau^{\prime \prime}=0$. If $\tau_{1}=0$, then substituting this and $\tau=0$ into (2.1) we obtain $q=0$, which together with $\sin \ell_{1} \xi_{1}=0$ yields $\sin \ell_{2} \xi_{2}=0$, substituting $\sin \ell_{1} \xi_{1}=\sin \ell_{2} \xi_{2}=0$ into (2.9) again gives us $\tau^{\prime \prime}=0$.

Lemma 3. Let $\tau(t, x)$, considered as a function of $x$ for an arbitrary but fixed value of $t$, have an extremum at $x=x_{0}$. Then $\tau\left(t, x_{0}\right) \leq 0$.

Proof. If $\sin \ell_{1} \xi_{1} \neq 0$ at $\left(t, x_{0}\right)$, then $\tau^{\prime}\left(t, x_{0}\right)=0$ and (2.11a) yields

$$
\tau=-\left(\frac{\sin \ell_{2} \xi_{2}}{\sin \ell_{1} \xi_{1}} \tau_{1}+q\right)^{2} \leq 0
$$

If $\sin \ell_{2} \xi_{2} \neq 0$ at $\left(t, x_{0}\right),(2.11 \mathrm{~b})$ yields the result.

Consider now the case $\sin \ell_{1} \xi_{1}=\sin \ell_{2} \xi_{2}=0$ at $\left(t, x_{0}\right)$. Substituting $\sin \ell_{1} \xi_{1}=$ $\sin \ell_{2} \xi_{2}=0$ into (2.8) and (2.9) we obtain $\tau^{\prime}\left(t, x_{0}\right)=\tau^{\prime \prime}\left(t, x_{0}\right)=0$. The fact that $x=x_{0}$ is an extremum then implies $\tau^{\prime \prime \prime}\left(t, x_{0}\right)=0$, which, using $(2.10)$, gives us $\ell_{1}^{2} \eta_{2}+\ell_{2}^{2} \eta_{1}=0$, 
with $\eta_{1}, \eta_{2}$ evaluated at $\left(t, x_{0}\right)$ according to (2.2). Then $\ell_{1}^{2} \eta_{2}+\ell_{2}^{2} \eta_{1}=0$ implies $\eta_{1} \eta_{2} \leq 0$. Substituting now $\sin \ell_{1} \xi_{1}=\sin \ell_{2} \xi_{2}=0$ into (2.1) we obtain $\tau=\eta_{1} \eta_{2} \leq 0$.

Lemma 4. Let $x_{0}$ be a local maximum of $\tau(t, x)$ considered as a function of $x$ for an arbitrary but fixed value of $t$. Then $\tau\left(t, x_{0}\right)<0$.

Proof. In view of Lemma 3 , it suffices to show that $\tau\left(t, x_{0}\right) \neq 0$. Assume $\tau\left(t, x_{0}\right)=0$ and consider

$$
f\left(\lambda_{1}, \lambda_{2}, t, x\right)=\left[\frac{\sin 2 \ell_{1} \xi_{1}}{2 \ell_{1}}-\left(x+12 \ell_{1}^{2} t-\lambda_{1}\right)\right]\left[\frac{\sin 2 \ell_{2} \xi_{2}}{2 \ell_{2}}-\left(x+12 \ell_{2}^{2} t-\lambda_{2}\right)\right]-q^{2} .
$$

Due to continuity of $f$ in all of its arguments, $f\left(\lambda_{1}, \lambda_{2}, t, x_{0}\right) \leq 0$ for $\lambda_{1}$ and $\lambda_{2}$ satisfying $\left|\lambda_{1}-p_{1}\right|+\left|\lambda_{2}-p_{2}\right|<\varepsilon$ for some sufficiently small $\varepsilon$. Thus $f\left(\lambda_{1}, \lambda_{2}, t, x_{0}\right)$ attains a local maximum as a function of $\lambda_{1}$ and $\lambda_{2}$ at $\lambda_{1}=p_{1}$ and $\lambda_{2}=p_{2}$ and therefore $\frac{\partial y}{\partial \lambda_{1}}=\tau_{2}=0, \frac{\partial f}{\partial \lambda_{2}}=\tau_{1}=0$ and the matrix $\left\|\frac{\partial f}{\partial \lambda_{1} \lambda_{1}}\right\|$ is nonnegative definite. On the other hand direct computations give us $\left\|\frac{\partial f}{\partial \lambda_{1} \partial \lambda_{j}}\right\|=\left(\begin{array}{ll}0 & 2 \\ 2 & 0\end{array}\right)$ which is not nonnegative definite. The obtained contradiction proves that the assumption $\tau\left(t, x_{0}\right)=0$ is false.

Lemma 5. Let $x_{0}$ be a local minimum of $\tau(t, x)$ (considered as a function of $x$ for an arbitrary but fixed value of $t)$ and $\tau\left(t, x_{0}\right)=0$. Then

$$
\tau_{1} \sin \ell_{2} \xi_{2}=\tau_{2} \sin \ell_{1} \xi_{1}=q \sin \ell_{1} \xi_{1} \sin \ell_{2} \xi_{2}=0 .
$$

Proof. Substituting $\tau^{\prime}=0$ into (2.8) we obtain

$$
\tau_{1} \sin ^{2} \ell_{2} \xi_{2}+\tau_{2} \sin ^{2} \ell_{1} \xi_{1}=-q \sin \ell_{1} \xi_{1} \sin \ell_{2} \xi_{2}
$$

Squaring both sides and replacing $\tau_{1} \tau_{2}$ with $q^{2}$ gives us

$$
\tau_{1}^{2} \sin ^{4} \ell_{2} \xi_{2}+\tau_{2}^{2} \sin ^{4} \ell_{1} \xi_{1}=-q^{2} \sin ^{2} \ell_{1} \xi_{1} \sin ^{2} \ell_{2} \xi_{2} .
$$

Since the left-hand side is nonnegative and the right-hand side is nonpositive, they both must be zero, yielding the result.

Lemma 6. Let $x_{0}$ be a local minimum of $\tau(t, x)$ (considered as a function of $x$ for an arbitrary but fixed value of $t$ ) and $\tau\left(t, x_{0}\right)=0$. Then either

$$
\tau_{1}\left(t, x_{0}\right)=\tau_{2}\left(t, x_{0}\right)=\sin \ell_{1} \xi_{1}=\sin \ell_{2} \xi_{2}=0
$$

or

$$
\tau_{1}\left(t, x_{0}\right)=\tau_{2}\left(t, x_{0}\right)=\cos \ell_{1} \xi_{1}=\cos \ell_{2} \xi_{2}=0 .
$$


Proof. By Lemma 5 one of (a) $\tau_{1}=\sin \ell_{1} \xi_{1}=0$, (b) $\tau_{2}=\sin \ell_{2} \xi_{2}=0$, (c) $\sin \ell_{1} \xi_{1}=$ $\sin \ell_{2} \xi_{2}=0$ or (d) $\tau_{1}=\tau_{2}=0$ must hold. Consider each case separately.

(a) $\tau_{1}=\sin \ell_{1} \xi_{1}=0$. Since $x_{0}$ is a local extremum and $\tau\left(t, x_{0}\right)=0$, Lemma 2 implies $\tau^{\prime \prime}=0$. Substituting $\tau_{1}=\sin \ell_{1} \xi_{1}=\tau^{\prime \prime}=0$ into (2.9) we obtain $\sin \ell_{2} \xi_{2}=0$. Again since $x_{0}$ is a local extremum and $\tau^{\prime \prime}=0$ we also have $\tau^{\prime \prime \prime}=0$, which along with (2.10) implies $\tau_{2}=0$.

(b) Similar to (a).

(c) $\sin \ell_{1} \xi_{1}=\sin \ell_{2} \xi_{2}=0$. Substituting these into (2.9) we obtain $\tau^{\prime \prime}=0$ which along with the fact that $x_{0}$ is an extremum yields $\tau^{\prime \prime \prime}=0$. Substituting $\tau^{\prime \prime \prime}=\sin \ell_{1} \xi_{1}=\sin \ell_{2} \xi_{2}=0$ into (2.10) gives us $\ell_{1}^{2} \tau_{2}+\ell_{2}^{2} \tau_{1}=0$ and therefore $\tau_{1} \tau_{2} \leq 0$. On the other hand substituting $\tau=0$ into (2.1) results in $\tau_{1} \tau_{2}=q^{2} \geq 0$ implying that either $\tau_{1}$ or $\tau_{2}$ is 0 . But $\ell_{1}^{2} \tau_{2}+\ell_{2}^{2} \tau_{1}=0$ and therefore once one of them vanishes so does the other one.

(d) $\tau_{1}=\tau_{2}=0$. Substituting $\tau_{1}=\tau_{2}=\tau=0$ into (2.1) we obtain $q=0$ and thus if either one of $\sin \ell_{1} \xi_{1}$ or $\sin \ell_{2} \xi_{2}$ is zero then so must the other one, yielding the result.

Let us now assume that neither $\sin \ell_{1} \xi_{1}$ nor $\sin \ell_{2} \xi_{2}$ vanish at $\left(t, x_{0}\right)$. Substituting $\tau=\tau_{2}=q=0$ into the expressions for $\tau^{\prime \prime \prime}$ and $\tau^{(4)}$ we obtain $\tau^{\prime \prime \prime}=\tau^{(4)}=0$. Since $x=x_{0}$ is an extremum we must have $\tau^{(\mathcal{S})}=0$ which combined with $\tau_{1}=\tau_{2}=q=0$ yields $\cos \ell_{1} \xi_{1}=\cos \ell_{2} \xi_{2}=0$.

Lemma 7. Let $x_{0}$ be a local minimum of $\tau\left(t_{0}, x\right)$ (considered as a function of $x$ ) and let $r\left(t_{0}, x_{0}\right)=0$. Then there exist two integers $n_{1}$ and $n_{2}$ either both even or both odd such that

$$
\begin{aligned}
& t_{0}=\frac{p_{1}-\gamma_{1}}{8 \ell_{1}^{2}}-\frac{\pi n_{1}}{16 \ell_{1}^{3}}=\frac{p_{2}-\gamma_{2}}{8 \ell_{2}^{2}}-\frac{\pi n_{2}}{16 \ell_{2}^{3}} \\
& x_{0}=\frac{3 \gamma_{1}-p_{1}}{2}+\frac{3 \pi n_{1}}{4 \ell_{1}}=\frac{3 \gamma_{2}-p_{2}}{2}+\frac{3 \pi n_{2}}{4 \ell_{2}}
\end{aligned}
$$

Proof. Lemma 6 implies that there exist two integers $n_{1}$ and $n_{2}$ either both even or both odd such that

$$
\begin{aligned}
& \left\{\begin{array}{l}
\eta_{1}=x_{0}+12 \ell_{1}^{2} t_{0}-p_{1}=0 \\
\xi_{1}=x_{0}+4 \ell_{1}^{2} t_{0}-\gamma_{1}=\frac{\pi n_{1}}{u_{1}}
\end{array}\right. \\
& \left\{\begin{array}{l}
\eta_{2}=x_{0}+12 \ell_{2}^{2} t_{0}-p_{2}=0 \\
\xi_{2}=x_{0}+4 \ell_{2}^{2} t_{0}-\gamma_{2}=\frac{\pi n_{2}}{u_{2}}
\end{array}\right.
\end{aligned}
$$

Solving the first system we obtain 


$$
t_{0}=\frac{p_{1}-\gamma_{1}}{8 \ell_{1}^{2}}-\frac{\pi n_{1}}{16 \ell_{1}^{3}}, \quad x_{0}=\frac{3 \gamma_{1}-p_{1}}{2}-\frac{3 \pi n_{1}}{4 \ell_{1}}
$$

whereas solving the second system we get

$$
t_{0}=\frac{p_{2}-\gamma_{2}}{8 \ell_{2}^{2}}-\frac{\pi n_{2}}{16 \ell_{2}^{2}}, \quad x_{0}=\frac{3 \gamma_{2}-p_{2}}{2}+\frac{3 \pi n_{2}}{4 \ell_{2}} .
$$

Proof of the Theorem. Part (a) If $\tau(t, x)$ had more than two zeros it would also have a nonnegative local maximum but that contradicts Lemma 4.

Part (b) Two zeros of the $\tau$-function merge into one if and only if for some $t_{0}$ $\tau\left(t_{0}, x\right)$ has a single zero $x_{0}$ which is also a local (as well as global) minimum. But according to Lemma 7 this can happen only if (2.12) holds. Vice versa if (2.12) holds then one can verify by direct computations that $\tau\left(t_{0}, x\right)$ has a local minimum at $x=x_{0}$ as well as $t\left(t_{0}, x_{0}\right)=0$. By solving (2.12) for $n_{1}$ and $n_{2}$ we obtain (1.7).

The order of zero at $x=x_{0}$ is easily verified by direct computations.

Acknowledgements. The authors would like to express their gratitude to the referee and Professor Lacey for their suggestions and corrections and to Ms. V. Spak for typing the manuscript.

\section{REFERENCES}

1. M. J. Ablowrtz and P. A. Clarkson, Solitons, Nonlinear Evolution Equations and Inverse Scattering (London Math. Society Lecture Notes), 149, Cambridge University Press, 1991).

2. S. CAenepeel and W. Malfliet, Internal structure of the two-soliton solutions of the KdV equation, Wave Motion 7 (1985), 299-305.

3. B. Fuchssteiner, The interaction equation, Phys. A 228 (1996), 189-211.

4. C. S. Gardner, J. M. Greene, M. D. Kruskal and R. M. Miura, Korteweg-de Vries equation and generalizations VI, Comm. Pure Appl. Math. 27 (1974), 97-133.

5. P. F. HodNETt and T. P. Moloney, On the structure during interaction of the two-soliton solution of the Korteweg-de Vries equation, SIAM J. Appl. Math. 49 (1989), 1174-1187.

6. H. Hu, Darboux transformation of Su-chain, in Proc. Conf. Diff. Geometry in honor of Prof. Su Buchin (1991, Shanghai, World Scientific Press, Singapore, 1993).

7. M. Kovalyov, Basic motions of the Korteweg-de Vries equation, in Nonlinear Analysis, Theory, Methods and Applications, to appear.

8. B. M. Levitan, Inverse Sturm-Liouville Problems (Chapter 6, VNU Science Press, 1987).

9. R. J. LeVeque, On the interaction of nearly equal solitons in the KdV equation, SIAM J. Appl. Math. 47 (1987), 254-262.

10. W. Malfliet and L. Van De Velde, A study of two interacting KdV solitons, Lett. Nuovo Cimento (2) 42 (1985), 179-183.

11. V. B. Matveev, Asymptotics of the multiposition-soliton $\tau$-function of the Kortewegde Vries equation and the supertransparency, J. Math. Phys. 35 (1994), 2955. 
12. V. B. Matveev, Phys. Lett. A. 135 (1992), 2009-2212.

13. T. P. Moloney and P. F. Hodnett, Soliton interaction (for the Korteweg-de Vries equation): a new perspective, J. Phys. $A 19$ (1986), L1129-L1135.

14. A. A. Stahlhofen, On completely transparent potentials of the Shrödinger equation, Phys. Rev. A51 (1995), 934.

15. A. Stahlmofen, Ann. der Physik 1 (1992), 554-569.

16. T. YonEYAMA, The Korteweg-de Vries two-soliton solution as interacting two single solitons, Progr. Theoret. Phys. 71 (1984), 843-846.

M. Kovalyov

Department of Mathematical Sciences

UNIVERSITY OF ALBERTA

Edmonton, Alberta

Canada T6G 2Gl
K. M. LEE

Department of Mathematics, Science and TECHNOLOGY

Heartland Community College

1226 Towanda AVenue

BLOOMINGTON

ILLINOIS 61701

USA 